\title{
A COMPARISON OF TOPICAL ANAESTHESIA AND RETROBULBAR BLOCK FOR CATARACT SURGERY
}

\author{
D. M. JOLLIFFE ${ }^{1}$, M. N. ABDEL-KHALEK ${ }^{2}$ and A. C. NORTON ${ }^{1}$ \\ Boston
}

\section{SUMMARY}

It is possible to remove a cataract and insert an intraocular lens under topical anaesthesia (lignocaine $4 \%$ preservative-free eye drops) with a van Lint block. This study was performed to evaluate this anaesthetic technique. Nineteen patients having cataract surgery with topical anaesthesia and a van Lint block were compared with 21 patients who received a retrobulbar block and a van Lint block. There were no significant differences between the two groups in patient characteristics, mean cardiovascular stress of the procedure, experience of pain during the operation or willingness to have the same anaesthetic technique again. Topical anaesthesia with a van Lint block is feasible for cataract surgery and is potentially safer than other regional anaesthetic techniques.

In an attempt to evaluate topical anaesthesia for cataract surgery, two groups of patients were studied prospectively and compared. One group had topical anaesthesia and the other had regional anaesthesia with a retrobulbar block. All patients also received a van Lint block. In all cases a recently developed modified surgical technique ${ }^{1}$ was used. The quality of anaesthesia was evaluated subjectively by asking the patients post-operatively about any pain or discomfort experienced in theatre. Patients were also asked whether they would agree to have the same anaesthetic technique if they were to require another eye operation. An objective comparison of the two techniques was made by quantifying the rate pressure product (RPP) at defined points in the procedure. As the RPP correlates with myocardial oxygen demand and cardiac work, the area under the RPP/time graph reflects the total cardiovascular stress. This indicator was compared between the groups.

From: Departments of ${ }^{1}$ Anaesthesia and ${ }^{2}$ Ophthalmology, Pilgrim Hospital, Boston, Lincolnshire, UK.

Correspondence to: M. N. Abdel-Khalek, Pilgrim Hospital, Boston, Lincolnshire PE21 9QS, UK.

\section{PATIENTS AND METHODS}

This study was approved by the local ethics committee.

\section{Patients}

Patients made the choice between general and local anaesthesia for their cataract surgery after discussion with the ophthalmic nurses. Those choosing local anaesthesia were studied prospectively but eyes which had been operated on before, for any reason, were excluded. A record was kept of the age, gender and ASA (American Society of Anesthesiologists) status of the patients. A patient who is ASA I is well and healthy, ASA II has mild to moderate systemic disease, ASA III has severe systemic disease that limits activity, or ASA IV has incapacitating systemic disease that is life-threatening.

\section{Anaesthesia}

Pre-operatively the patients were not starved and after assessment were all given oral diazepam $5 \mathrm{mg}$, promethazine hydrochloride $10 \mathrm{mg}$ and chlorpromazine hydrochloride $10 \mathrm{mg}$, an hour and a half before surgery. The eyes to be operated on were prepared with a standard regimen of mydriatic and antibiotic eye drops.

In the anaesthetic room ECG, non-invasive blood pressure (Dinamap) and pulse oximetry monitoring were established. Venous access was secured in the dorsum of the left hand using a 20 gauge intravenous cannula inserted without local anaesthesia. Patients were randomised by year of birth. Those born in even years received topical anaesthesia with $0.5 \mathrm{ml}$ preservative-free $4 \%$ lignocaine eye drops. After instillation of the drops into the conjunctival sac, the eyelids were held apart for about a minute to maximise contact between the local anaesthetic solution and the corneal and conjunctival surfaces and to soak the nerve endings. Patients born in odd years received a retrobulbar block using $3 \mathrm{ml}$ of a 10 $\mathrm{ml}$ mixture containing $7 \mathrm{ml} 0.75 \%$ bupivacaine, $3 \mathrm{ml}$ 
$2 \%$ lignocaine and 1500 IU hyalase. All patients received a zygomatic nerve block (van Lint) with 2 $\mathrm{ml} 2 \%$ lignocaine injected deep along the upper and lower orbital rims and along the superior edge of the zygomatic arch. No injection was given to fixate the superior rectus muscle. All regional anaesthesia was given by the surgeon (M.A.-K.) as this promotes the best chance of observing an orbital haemorrhage or other complication before draping the patient and incising the eye. ${ }^{2}$

In theatre monitoring of the patient's ECG, blood pressure and pulse oximetry were continued and all patients received supplemental oxygen. During the procedure any arrhythmias were noted.

\section{Surgery}

All the operations were performed by the same surgeon (M.A.-K.) using the same technique. ${ }^{1}$ This modified technique enables a cataract to be extracted and an intraocular lens inserted under topical anaesthesia. The effectiveness of the van Lint block in reducing the strength of the palpebral reflex was assessed by the ease with which a Barraquer's onepiece wire speculum was inserted. A corneo-limbal incision from 10 o'clock to 2 o'clock was made with a diamond knife in a two-step fashion. Firstly a perpendicular incision in the superficial half of the cornea was made and then a deep slanting incision towards the pupil to enter the anterior chamber. A wide 'can-opener' capsulotomy was used to allow easy delivery of the nucleus and avoid pain caused by stretching the zonules in the ciliary body. Phacoemulsification of the nucleus was not employed. The equatorial area of the lens bag was flushed with balanced salt solution through a curved $26 \mathrm{G}$ lacrimal needle. This 'equatorial flush' helped to break the attachments of the lens fibres and enabled the lens cortex to be rapidly aspirated. A rigid $12.00 \mathrm{~mm}$ diameter intraocular lens implant was inserted into the capsular bag and the corneo-limbal incision was sutured closed with 10/0 continuous nylon. At the end of the operation a subconjunctival injection of gentamicin and betamethasone was given.

\section{Assessment}

All the assessments were made by the same observer (D.M.J.). Baseline measurements of each patient's heart rate and systolic, mean and diastolic blood pressures were made after their arrival in the anaesthetic room (1). These readings were repeated after insertion of the venous cannula (2), instillation of eye drops (in the topical anaesthesia group) or insertion of the retrobulbar block needle (3), and after the van Lint block was performed (4). On arriving in theatre further readings of the patient's heart rate, systolic, mean and diastolic blood pressures were taken (5), and these were repeated when the superior rectus suture was inserted (6), the nucleus delivered (7), the lens implanted (8), the corneo-limbal incision sutured (9) and the subconjunctival injection given (10). For each patient the measurements of heart rate and systolic blood pressure were used to calculate the rate pressure product (RPP) at the 10 defined events points during the procedure. The timing of these measurements reflected either baseline conditions $(1,5)$ or points in the procedure when the patient was most likely to feel pain. The area under the curve $(\mathrm{AuC})$ of RPP ( $y$-axis) plotted against time ( $x$-axis) was then calculated for each patient.

Post-operatively on the ward all the patients were interviewed by the anaesthetist and asked whether they had experienced any pain or discomfort in theatre (i.e. after the regional anaesthesia had been instituted). Responses were graded on a five-point scale: 0 , no feeling; 1 , aware of the steps of the procedure; 2, discomfort; 3, tolerable pain; 4, intolerable pain. Satisfaction was assessed by asking whether the patient would choose to have the same anaesthetic technique again should they require another eye operation. Any comments were noted.

\section{Statistics}

In planning the study, the authors concluded that a $30 \%$ difference in mean RPP between the two groups would constitute a clinically important difference. The sample size for each study group was estimated by a comparison of two means method ${ }^{3}$ assuming an $80 \%$ power of detecting a $30 \%$ difference in mean RPP assuming a standard deviation of 3000 in RPP calculations in each group. This method estimated a sample size of 19 patients in each group.

RPP data were analysed according to the method of Matthews et al. ${ }^{4}$ for the analysis of serial measurements in biomedical research. The AuC calculations as the summary measure were subjected to significance testing with one-way analysis of variance (ANOVA). Responses to patient assessments of intra-operative pain and willingness to undergo the procedure again were analysed using chi-squared $\left(\chi^{2}\right)$ tests with given degrees of freedom (d.f.).

\section{RESULTS}

Initially 44 patients were included in the study but 4 were subsequently excluded from the analysis. In each group one patient was excluded because the lens was inserted into the anterior chamber. This is a more painful procedure than insertion into the posterior chamber because iridectomy is necessary. Two other patients who received topical anaesthesia were excluded, one for anaesthetic and one for surgical reasons. One patient complained of 
Table I. Patient characteristics

\begin{tabular}{lcc}
\hline & $\begin{array}{c}\text { Retrobulbar } \\
(n=21)\end{array}$ & $\begin{array}{c}\text { Local drops } \\
(n=19)\end{array}$ \\
\hline Mean age (range) & $77.8(67-93)$ & $78.1(70-91)$ \\
Gender M:F & $7: 14$ & $7: 12$ \\
ASA status I:II:III & $3: 15: 3$ & $2: 12: 5$ \\
\hline
\end{tabular}

Table II. Total time (minutes) for anaesthesia and cataract surgery

\begin{tabular}{lcc}
\hline & Retrobulbar & Local drops \\
\hline Mean \pm SD & $32.67 \pm 6.55$ & $33.47 \pm 5.52$ \\
Range & $23-50$ & $24-42$ \\
\hline
\end{tabular}

intolerable pain on insertion of the superior rectus suture. As this was not relieved by additional local anaesthetic eye drops the technique was abandoned and he was given a retrobulbar block. He remained extremely tense and received intravenous hydralazine to control systolic hypertension. With hindsight this patient would have been better managed with a general anaesthetic. In the other case that was excluded from the analysis, removal of the cataract was complicated by an expulsive haemorrhage. The eye was closed with difficulty without inserting the lens. Supplementary anaesthesia with additional local anaesthetic eye drops was given.

Thus 40 patients were included in the analysis. Twenty-one received a retrobulbar block and 19 topical local anaesthesia. Their characteristics are given in Table I. The total time taken for anaesthesia to be instituted and surgery performed is shown in Table II and was very similar for the two groups.

Fig. 1 is an example of one patient's heart rate and systolic blood pressure plotted against time. At the 10 defined events during the procedure these measurements were used to calculate RPP which was then plotted against time, as shown in Fig. 2. The area under this 'curve' $(\mathrm{AuC})$ was calculated for each patient. It reflects the total cardiovascular stress of the procedure.

Table III shows the mean AuC of the RPP/time graphs for each group. There is no significant difference between these (using one-way ANOVA),

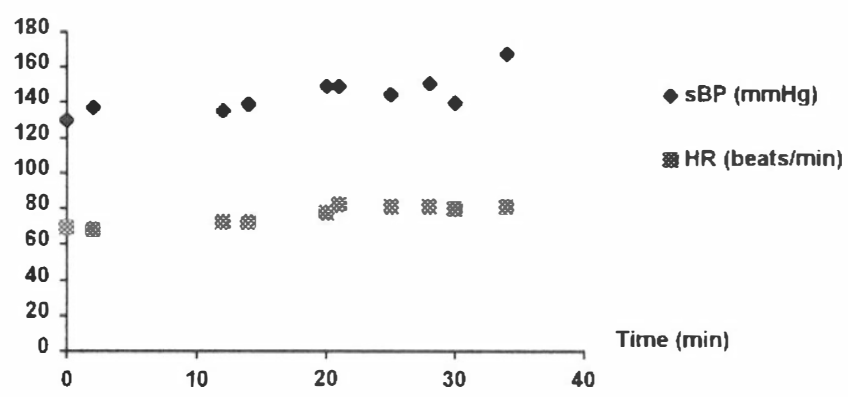

Fig. 1. Graph of one patient's systolic blood pressure $(s B P)$ and heart rate $(H R)$ against time.

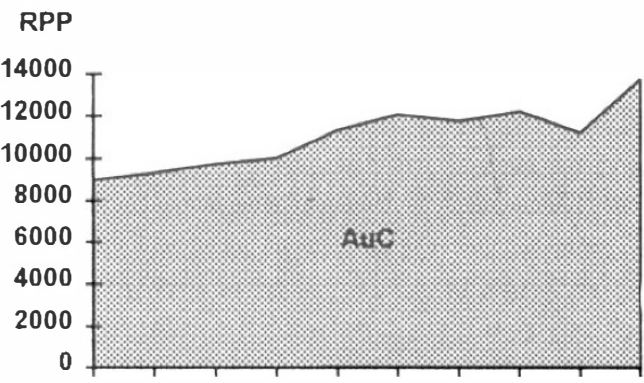

Time (min)

Fig. 2. Graph of rate pressure product (RPP) versus time.

so the total cardiovascular stress is not significantly different between the two techniques.

Table IV documents the patients' post-operative rating of the degree of feeling or pain during the surgical procedure. Although there were more patients who experienced tolerable pain in the topical anaesthesia group, this was not statistically significant.

The majority of patients were satisfied with the anaesthetic technique they received for cataract surgery and were willing to have the same technique again (Table V). The two patients who would not agree to repeat topical anaesthesia gave the pain of insertion of the van Lint block as their reason. In all cases orbicularis action was abolished by the van Lint block.

No significant arrhythmia was noted during the study.

\section{DISCUSSION}

In 1884 Knapp reported the successful extraction of a cataract using topical anaesthesia provided by frequent drops of $5 \%$ cocaine..$^{5}$ Today various

Table III. Mean AuC of the RPP/time graphs

\begin{tabular}{ll}
\hline Retrobulbar (mean \pm 1 SD) & $310165 \pm 103119$ \\
Local drops (mean \pm 1 SD) & $333936 \pm 104194$ \\
\hline
\end{tabular}

Table IV. Patients' post-operative rating of feeling or pain during the procedure

\begin{tabular}{lcc}
\hline & Retrobulbar & Local drops \\
\hline No feeling & 10 & 6 \\
Aware of steps of procedure & 7 & 7 \\
Discomfort & 3 & 2 \\
Tolerable pain & 1 & 4 \\
Intolerable pain & 0 & 0 \\
\hline$\chi^{2}=2.90$, d.f. $=3$. No significant difference between the groups.
\end{tabular}

Table V. Patient willingness to have same anaesthetic technique again

\begin{tabular}{lcc}
\hline & Retrobulbar & Local drops \\
\hline Yes & 19 & 16 \\
No & 1 & 2 \\
Don't know/No memory & 1 & 1 \\
\hline$\chi^{2}=0.49$, d.f. $=2$. No significant difference between the groups.
\end{tabular}


general and regional anaesthetic techniques are used for cataract surgery, including retrobulbar, peribulbar, sub-Tenon's and subconjunctival application of local anaesthetic. We chose to compare topical anaesthesia with retrobulbar anaesthesia because the latter is a standard with which other techniques have been compared and it is highly effective in providing analgesia, akinesia and optimal orbital pressure. $^{6}$

Topical anaesthesia (given as eye drops) could not be simpler to administer and if analgesia is insufficient, or the surgery is prolonged, additional anaesthesia (extra eye drops), can easily be given. This route of administration avoids inserting a needle into the eye and injecting local anaesthetic into potentially dangerous places, thereby avoiding all the possible sight- and life-threatening complications of other regional anaesthetic techniques. ${ }^{7}$

However, in addition to being safe, a feasible anaesthetic technique must allow the surgeon to perform the operation and be acceptable to the patient. It should cause minimal discomfort to the patient and the least delay for the surgeon.

Topical anaesthesia does not provide complete akinesia and residual extraocular muscle function allows movement of the globe during surgery and may increase the intraocular pressure. This can be reduced by having a relaxed and co-operative patient. A superior rectus bridle suture will steady the globe in the vertical plane but the surgeon must be able to tolerate some movement, which will be exaggerated by the magnification of the microscope. A van Lint block of the facial nerve was performed in all patients to paralyse the orbicularis muscle and so prevent squeezing of the eyelids against the speculum which increases the intraocular pressure.

The surgeon may have to cope with unexpected events. In this study topical anaesthesia provided sufficient analgesia to insert the lens implant into the anterior chamber in one case and to close an eye with an expulsive haemorrhage. If the eye is open, conversion to a general anaesthetic is not an option but another regional technique may be used. One of our patients, who was subsequently excluded from the analysis, received topical anaesthesia and was then given a retrobulbar block.

General anaesthesia has a higher incidence of post-operative nausea and sore throat and a longer time until the resumption of oral intake than local anaesthesia for cataract surgery. ${ }^{8}$ Our patients were often aware of the advantages of avoiding general anaesthesia but worried that if they were awake they might have pain during surgery and/or be able to see the procedure. Anxiolytic premedication may be helpful. In this study an oral cocktail of sedatives was given to all patients as this was the routine ward practice. However, the best anxiolytic is confidence in the staff and good rapport with a surgeon who is willing to talk to the patient during their operation. ${ }^{9}$

In this study more patients with topical anaesthesia experienced tolerable pain than those with a retrobulbar block. This was not statistically significant, and was not associated with a statistically significant increase in the average cardiovascular stress of the procedure. Thus, topical anaesthesia is suitable for use in patients with borderline cardiovascular reserve (ASA IV), although such patients may have difficulty lying flat. An attempt to score pain using a visual analogue scale was abandoned because individuals' scores did not correlate at all with their verbal descriptions. Two patients were not willing to have topical anaesthesia again and both gave the pain of inserting the van Lint block as the reason for this. A van Lint block may not be necessary. Cataract extraction has been successfully performed without a facial nerve block, using $1 \%$ amethocaine eye drops and a subconjunctival injection of $1 \mathrm{ml}$ of $2 \%$ lignocaine. ${ }^{10}$

As topical anaesthesia does not block the ciliary body, patients will be able to perceive light and would be anticipated to have pain with procedures that involve pressure or distortion of the eye. However, no patient remarked on the bright light of the operating microscope and analgesia was adequate for insertion of a lens into the anterior chamber in one patient and to close the eye with an expulsive haemorrhage.

Increasingly cataract surgery is being performed as a day case procedure. Phacoemulsification (ultrasound fragmentation of the lens) is being used to remove cataracts and foldable intraocular lens implants are being inserted through smaller incisions which do not require suturing. ${ }^{11}$ Cost will probibit these advances in developed countries but topical anaesthesia may prove to be the ideal anaesthetic technique in both situations. ${ }^{12}$ It is certainly the simplest to administer and provides feasible operating conditions. It is acceptable to most patients and has the advantages of regional anaesthesia without the potential complications of inserting a needle and injecting local anaesthetic into the eye. Safety is a particularly important consideration when there is no anaesthetist to monitor and resuscitate the patient.

Key words: Cataract surgery, Topical anaesthesia, Retrobulbar anaesthesia.

\section{REFERENCES}

1. Abdel-Khalek MN, Refaat RF. Surgical modifications required for planned extracapsular cataract extraction under topical anaesthesia with van Lint block. Eur J Implant Refract Surg 1995;7:338-41.

2. Liu C, Redmond R. Choice in local anaesthesia. Br J Ophthalmol 1993;77:758. 
3. Kirkwood BR. Essentials of medical statistics. Oxford: Blackwell Scientific, 1988:196.

4. Matthews JNS, Altman DG, Campbell MJ, Royston P. Analysis of serial measurements in medical research. BMJ 1990;300:230-5.

5. Stevens JD. A new local anaesthesia technique for cataract extraction by one quadrant sub-Tenon's infiltration. Br J Ophthalmol 1992;76:670-4.

6. Wong DH. Regional anaesthesia for intraocular surgery. Can J Anaesth 1993;40:635-57.

7. Rubin AP. Complications of local anaesthesia for ophthalmic surgery. Br J Anaesth 1995;75:93-6.
8. Barker JP, Vafidis GC, Hall GM. Postoperative morbidity following cataract surgery: a comparison of local and general anaesthesia. Anaesthesia 1996;51: 435-7.

9. Hamilton RC. Techniques of orbital regional anaesthesia. Br J Anaesth 1995;75:88-92.

10. Smith R. Cataract extraction without retrobulbar anaesthetic injection. Br J Ophthalmol 1990;74:205-7.

11. Towler HMA, Lightman S. Recent advances in ophthalmology. BMJ 1996;312:889-92.

12. Forrester JV. Local anaesthesia for eye surgery. Br J Ophthalmol 1992;76:705. 